\title{
Novedades nomenclaturales para Gaultheria (Ericaceae) en Chile
}

\section{Novelties on Gaultheria (Ericaceae) in Chile}

\author{
Sebastián Teillier ${ }^{1 *}$, Roberto Rodríguez ${ }^{2} \&$ Peter W. Fritsch ${ }^{3}$ \\ ${ }^{1}$ Escuela de Arquitectura del Paisaje, Universidad Central de Santiago de Chile, Santa Isabel 1160, Santiago. Chile. \\ 2Departamento de Botánica, Facultad de Ciencias Naturales y Oceanográficas, Universidad de Concepción, Casilla 160-C, \\ Concepción, Chile. \\ 32Department of Botany, California Academy of Sciences, San Francisco, California 94118, USA. \\ *steillier@gmail.com
}

\begin{abstract}
RESUMEN
Una actualización de Gaultheria en el marco del proyecto "Flora de Chile" nos ha llevado a proponer dos nuevas combinaciones y una lectotipificación para este género. Pernettya linifolia Phil. fue descrita por R.A. Philippi, y posteriormente incluida, ya sea en Gaultheria (Pernettya) mucronata o en Gaultheria (Pernettya) poeppigii; en este trabajo se propone que se trata de una buena especie, que de acuerdo con la nomenclatura actual de las ericáceas, debería denominarse Gaultheria linifolia (Phil.) Teillier \& R.A. Rodr. La combinación Gaultheria angustifolia (Lindl.) Teillier fue recientemente publicada para otra especie de Chile y Argentina, pero se demostró que el nombre constituye un homónimo posterior a Gaultheria angustifolia Brandegee, una especie mexicana; en este trabajo se propone su reemplazo por Gaultheria marticorenae Teillier \& P.W. Fritsch. Finalmente, se propone un lectotipo para el sinónimo heterotípico Pernettya leucocarpa var. linearis Reiche.
\end{abstract}

Palabras clave: Flora de Chile, Gaultheria, Pernettya, Ericaceae.

\begin{abstract}
After a revision of Gaultheria species for Flora de Chile project, we found new specimens and information that has prompted us to make two new combinations and a lectotype for this genus. Pernettya linifolia Phil. was described by R. A. Philippi and subsequently treated taxonomists as a variety of either Gaultheria (Pernettya) mucronata or Gaultheria (Pernettya) poeppigii. We treat Pernettya linifolia at the species level, and move it to Gaultheria as Gaultheria linifolia (Phil.) Teillier \& R.A. Rodr., in line with the new classification of Ericaceae. The combination Gaultheria angustifolia (Lindl.) Teillier (Ericaceae) was recently made for a distinct species from Chile and Argentina, but this name is a later homonym of Gaultheria angustifolia Brandegee from Mexico. A new name, Gaultheria marticorenae Teillier \& P.W. Fritsch, is therefore provided as a replacement for this species. A lectotype for the heterotypic synonym Pernettya leucocarpa var. linearis Reiche is also designated.
\end{abstract}

KeYwords: Flora of Chile, Gaultheria, Pernettya, Ericaceae.

\section{INTRODUCCIÓN}

Gaultheria L., tal como se entiende hoy en día, comprende tanto las especies descritas bajo Gaultheria, como las descritas como Pernettya Gaudich., dado que según Middleton \& Wilcock (1990) y Bush et al. (2009), las diferencias al nivel de la morfología de los frutos no serían suficientes para mantenerlos como géneros separados, siendo prioritario el primero de ellos.

En el marco de la revisión de materiales destinados al estudio de Gaultheria para el proyecto "Flora de Chile", cuya monografía fue publicada por Teillier \& Escobar (2013), se hicieron combinaciones nuevas y quedaron por resolver algunas dudas respecto de taxones cuyos ejemplares tipo no presentaban suficiente información para ser incluidos en alguna de las doce especies que se concluyó forman este género en Chile. Producto de esa misma publicación otros especialistas y personas interesadas en la botánica de campo han interactuado con los autores para comunicarles novedades que complementan dicha publicación y aportan datos relevantes para producir una mejor revisión del género para el proyecto. 
Producto de dichos aportes y sugerencias, los objetivos de este artículo son: establecer la ubicación de Pernettya linifolia Phil., un taxón que quedó como "incertae sedis" en la monografía; proponer un cambio de nombre para Gaultheria angustifolia (Lindl.) Teillier, por existir un nombre que la antecede; y proponer un lectotipo para Pernettya leucocarpa DC. var. linearis Reiche.

\section{Gaultheria linifolia (Phil.) Teillier \& R. A. Rodr. (ERicaceae)}

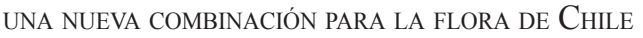

Entre las especies descritas por R. A. Philippi (1864), se encuentra Pernettya linifolia Phil., cuyo tipo fue recolectado por R. Pearce posiblemente entre las localidades de Corral y Río Bueno. Por las características del fruto, una baya, fue asignada a Pernettya, pero especialistas posteriores reasignaron su rango a variedad; primero Reiche (1905), de Gaultheria (Pernettya) mucronata (L.f.) Hook. et Arn., y luego Kausel (1949) y Sleumer (1985), de G. poeppigii DC., en todos los casos es posible que no hayan contado más que con el material tipo para efectuar dicha asignación. Dadas las dudas que existían, en la revisión de Teillier \& Escobar (2013) el taxon fue dejado como incertae sedis.

Posterior a la revisión se ha descubierto una población con rasgos morfológicos similares a los del ejemplar tipo en la cordillera de la Costa de la provincia de La Unión, cuyas características permiten reunirla con colecciones anteriores efectuadas entre las provincias de Valdivia y Chiloé, siendo la característica principal del grupo la presencia de hojas lineares, de 1-2 $\mathrm{mm}$ de ancho.

\section{DESCRIPCIÓN}

Gaultheria linifolia (Phil.) Teillier \& R. A. Rodr. comb.nov.

Basiónimo: Pernettya linifolia Phil., Linnaea 33: 172. 1864. Typus: "In montibus inter Corral et ostium fluminis Riobueno detexit orn. R. Pearce.” (holotypus SGO-53715).

Pernettya mucronata (L. f.) Gaudich. ex Spreng. var. linifolia (Phil.) Reiche, Anales Univ. Chile 117: 491.1905; Pernettya prostrata (Cav.) DC. var. linifolia (Phil.) Sleumer, Notizbl. Bot. Gart. Berlin-Dahlem 13: 211.1936; Pernettya myrtilloides Zucc. ex Steud. var. linifolia (Phil.) Kausel, Revista Univ. (Santiago) 34: 164.1949; Pernettya poeppigii (DC.) Klotzsch var. linifolia (Phil.) Sleumer, Lilloa 25: 550.1951; Gaultheria poeppigii DC. var. linifolia (Phil.) D.J. Middleton, Edinburgh J. Bot. 47 (3): 298.1990.

Arbusto siempreverde, de 20-70 cm de alto. Hojas brevemente pecioladas, glabras, alternas, simples, lineares, de 1-1,5 x 0,1$0,2 \mathrm{~cm}$, enteras a ligeramente dentadas, nervadura central bien marcada; en algunos ejemplares las hojas son casi perpendiculares al tallo, en otros, forman un ángulo agudo.
Flores solitarias, axilares, sobre pedicelos curvados, de 5-7 $\mathrm{mm}$ de longitud, con 4 bractéolas y dos brácteas pequeñas en su base. Cáliz 5-partido, parte libre de los sépalos de 1-1,5 mm de longitud; corola gamopétala, urceolada, con 5 pétalos blancos, de 4 a 4,5 mm de longitud; androceo con 10 estambres de 2 $\mathrm{mm}$ de longitud; ovario súpero, globoso y piriforme. Fruto, una baya, que de acuerdo con la descripción de R. A. Philippi, sería de color blanco, coronada por el estilo.

\section{DisTRIBUCIÓN GEOGRÁFICA}

Especie endémica del bosque templado de la cordillera de la Costa de Chile, se la encuentra entre la Región de Los Ríos, Reserva Nacional Valdivia, río Chaihuín, y la Región de Los Lagos, provincia de Chiloé, cordillera de Piuchén.

\section{OBSERVACIONES}

La estrechez de sus hojas, siempre lineares, distingue a esta especie de sus más cercanas morfológicamente, G. poeppigii y G. marticorenae, esta última suele tener, además, un mucrón apical.

El material examinado contiene sólo flores marchitas por lo que se ha dificultado la descripción de la sexualidad de la especie, en Gaultheria existen especies con flores hermafroditas y unisexuales, en el último caso las especies suelen ser dioicas.

Material estudiado: Chile, Prov. Ranco, P. N. Alerce Costero, estero Lañihual, 20-II-2014, A. Zúñiga 232 (CONC 179061). Región de Los Lagos, prov. Chiloé, cordillera del Piuchén por Piruquina, 28-X-1970, R. Gajardo y A. Troncoso s/n (CONC 59094); cordillera de San Pedro por Piruquina, 26-X a 5-XI-1970, G. Martínez s/n (CONC 59116); Isla Grande de Chiloé, cordillera del Piuchén, río Abtao, $500 \mathrm{~m}$ s.n.m., Villagrán 4926, 15-II-1983 (CONC 55777); TrumaoChiloé, cerca de Los Molinos del río Grande, 1-VI-1932, C. Junge (CONC 2440).

Se incluye en el material estudiado un ejemplar fotografiado y subido a Internet, que se ha atribuido a Gaultheria linifolia, la fotografía se tomó en la Reserva Nacional Valdivia, cerca del río Chaihuín (https://www.flickr.com/photos/fjbn/4832647317/).

\section{ECOLOGÍA Y ESTADO DE CONSERVACIÓN}

Las colecciones conocidas de la especie se encuentran en las partes medias y altas de la cordillera de la Costa entre las regiones de Los Ríos y de Los Lagos; de acuerdo con la propuesta de Luebert \& Pliscoff (2006), con base en la única referencia de altitud existente, $500 \mathrm{~m}$, en Chiloé (ejemplar de Villagrán, 4926), se propone que la especie formaría parte de la vegetación de los pisos del bosque laurifolio templado costero de Weinmannia trichosperma Cav. y Laureliopsis philippiana (Looser) Schodde ubicado entre 0-700 m de altitud en la cordillera Pelada y entre $0-500 \mathrm{~m}$ en la cordillera del Piuchén y del piso del bosque resinoso templado costero 
de Fitzroya cupressoides (Molina) I.M. Johnst., distribuido en las cordilleras Pelada, del Sarao y Piuchén (San Pedro). Tres de las colecciones, de acuerdo con la información de los recolectores, están asociadas a cursos de agua, por lo que podría tratarse de una especie higrófila de quebrada o de borde de río.

Si bien por lo escaso del material conocido resulta imposible aplicar todos los criterios UICN de clasificación de especies en categorías de amenaza (UICN 2001), los datos que se dispone apuntan a que se trata de una especie con una extensión de su área de unos $350 \mathrm{~km}$ lineales, sin embargo, la especie debe ubicarse sólo en los sitios donde la cordillera de la Costa alcanza dimensiones de macizo: cordilleras Pelada, Sarao y Piuchén (San Pedro), por lo que el área efectiva de ocupación debe ser bastante más restringida; otro indicador de escasez corresponde a que existen muy pocas colecciones, la del tipo (posiblemente de la cordillera Pelada), una reciente (2014) también de la cordillera Pelada, y tres en la cordillera de Piuchén, en Chiloé. Posiblemente se trate de una especie "vulnerable", ya que a su escasez, se suma que crece en localidades que no han estado al margen de las actividades humanas, sumándose a la tradicional explotación maderera, la sustitución por árboles introducidos y la instalación de proyectos de parques eólicos.

Clave para distinguir a $G$. linifolia de las especies simpátricas con fruto tipo baya (Basada en Teillier \& Escobar 2013):

1. Flores dispuestas en inflorescencias multifloras. Hojas de 5-9 $\mathrm{cm}$ de longitud.

G. insana

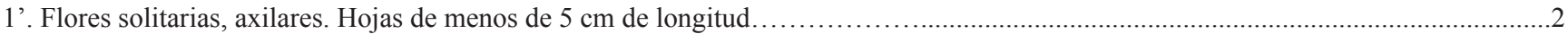

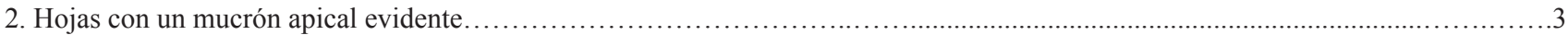

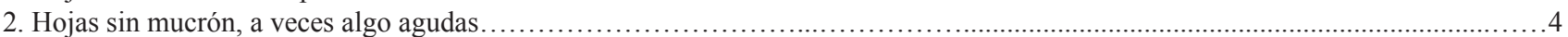

3. Hojas aovadas a aovado-oblongas, $4-10 \mathrm{~mm}$ ancho; mucrón siempre rígido..........................................G. mucronata

3'. Hojas lanceoladas a lineares, $2-3 \mathrm{~mm}$ de ancho; mucrón rígido a débil.............................................................G. marticorenae

4. Hojas oblongas a elípticas de 3-5 $\mathrm{mm}$ de ancho.... G. poeppigii

4'. Hojas estrictamente lineares, de 1-2 $\mathrm{mm}$ de ancho.

G. linifolia

Gaultheria marticorenae Teillier \& P.W. Fritsch, un NOMBRE NUEVO PARA GAULTHERIA ANGUSTIFOLIA (LiNDL.) Teillier, y un LeCtotipo Para Pernettya LeUCocarpa DC. VAR. LINEARIS REICHE

En la reciente revisión Gaultheria (Ericaceae) de Chile para el proyecto "Flora de Chile" (Teillier \& Escobar, 2013) se publicó el nombre Gaultheria angustifolia (Lindl.) Teillier para denominar a una especie arbustiva de Gaultheria con un fruto carnoso tipo baya, muy afín a $G$. mucronata (L. f.) Hook. et Arn., que había sido descrita por Lindley en 1840 bajo Pernettya angustifolia Lindl. y a la que se consideró, desde un punto de vista morfológico y fitogeográfico, como una buena especie.

Gaultheria angustifolia (Lindl.) Teillier resultó ser un homónimo posterior de Gaultheria angustifolia Brandegee, Zoe 5: 251. 1908, una especie mexicana (holotipo: Purpus 2040 UC 125366, imagen on line; Luteyn, 1995), por lo que se propone un nuevo nombre para esa entidad.

Gaultheria marticorenae Teillier \& P.W. Fritsch, nom. nov. Nombre reemplazado: Gaultheria angustifolia (Lindl.) Teillier, Gayana Bot. 70 (1): 138. 2013, non Gaultheria angustifolia Brandegee, Zoe 5: 251. 1908.

Basiónimo: Pernettya angustifolia Lindl., Bot. Reg. 26: tab. 63. 1840. Pernettya mucronata (L.f.) Gaudich. ex Spreng. var. angustifolia (Lindl.) Reiche, Anales Univ. Chile 117: 491. 1906. Gaultheria mucronata (L.f.) Hook. var. angustifolia (Lindl.) D.J. Middleton, Edinburgh J. Bot. 47(3): 298. 1990. TIPO: Ex hortus, Lindley anno 1840, cult. Birmingham Botanical Garden (holotipo: K 780478 imagen online; isotipo: BR 699709 (imagen on line).

Pernettya leucocarpa DC. var. linearis Reiche. Anales Univ. Chile 117: 494. 1906. TIPO: "ñadi de Puyehue", Januario 1905, K.F. Reiche s.n. (lectotipo aquí designado: SGO 3783 imagen on line).

Reiche (1905) cita dos especímenes en el protólogo de Pernettya leucocarpa DC. var. linearis Reiche: "cordilleras de Chillan" and "ñadi de Puyehue". El primero de ellos aparentemente con la etiqueta "Primera muda desde los Baños de Chillán, Janr 1877" (SGO 3784 imagen online) corresponde a una colección de R. A. Philippi s.n. anotada por Reiche como Pernettya leucocarpa DC. var. linearis Reiche (el apellido del autor esta signado en la etiqueta con una "R") y el último, tiene una etiqueta del mismo K. F. Reiche s.n. con la localidad de "ñadi de Puyehue", January 1905 (SGO 3783 imagen on line). Los especímenes fueron anotados como "tipo" y "cotipo" de respectivamente $P$. leucocarpa var. linearis por C. Muñoz P. en marzo de 1946, pero ambos, de a acuerdo con el protólogo de Reiche serían "sintipos" de Pernettya leucocarpa var. linearis.

Se ha seleccionado como lectotipo el espécimen de Reiche por haber sido coleccionado y anotado de mano del propio Reiche, considerando que para las plantas chilenas SGO es, además, el herbario que alberga las colecciones en 
Chile de dicho botánico. No hemos encontrado duplicados de esa colección. Respecto del ejemplar coleccionado en los Baños de Chillán por R.A. Philippi, nos parece que debe atribuirse a Gaultheria poeppigii DC.

\section{DESCRIPCIÓN}

Arbusto de 20-100 cm de altura. Hojas glabras, discolores, con las nervaduras principales fuertemente marcadas en el envés, cortamente pecioladas, lineares, a lanceoladoelípticas u oblongas, de 12-15 (18) x ca. $3 \mathrm{~mm}$, borde aserrado, los dientes muy agudos hasta mucronulados; con un pequeño reborde, muy atenuadas en el ápice y en la base, de consistencia poco rígida y mucrón breve. Especie dioica. Flores unisexuales, solitarias, axilares, pedicelo de 4-8 $\mathrm{mm}$. Cáliz 5-partido, lóbulos de $2 \mathrm{~mm}$ angostamente triangulares, algo agudos. Flores masculinas con una corola campanulada de $c a .5 \mathrm{~mm}$; androceo con 10 estambres, anteras de $1 \mathrm{~mm}$, filamentos de 1,2-1,5 mm; gineceo con ovario de 1,2 $\mathrm{mm}$, piriforme, estéril. Flores femeninas con una corola de $c a$. $4 \mathrm{~mm}$, urceolado-cilíndrica; estambres estériles; gineceo con ovario fértil, estigma con 5 lóbulos bien desarrollados. Fruto, una baya de 6-8 (10) mm de diámetro, deprimida en la base y el ápice, casi globosa, rosada, cáliz ni acrescente ni carnoso.

DistribuCión GEOGRÁfica: Especie nativa de Chile y Argentina. En Chile tiene una distribución disyunta, encontrándose una población aislada y con carácter relicto en la localidad de Fray Jorge (Región de Coquimbo) y luego, desde la provincia de Arauco hasta la de Chiloé; es frecuente en sitios húmedos incluso en los suelos anegados de los ñadis y pantanos (Kausel 1949).

Etimología: Este nombre está dedicado a Clodomiro Marticorena (1929-2013), profesor de botánica de la Universidad de Concepción, uno de los principales botánicos de Chile de este siglo.

\section{AGRADECIMIENTOS}

Al Sr. Alberto Zúñiga por poner atención a G. linifolia y proveer gentilmente material para el herbario del Departamento de Botánica de la Universidad de Concepción. A Socorro González por confirmar la existencia del homónimo en la flora de México.

\section{BIBLIOGRAFÍA}

Bush, C.M., L. Lu, P.W. Fritsch, D.Z. Li \& K.A. Kron. 2009. Phylogeny of Gaultherieae (Ericaceae: Vaccinoideae) based on DNA sequence data from matK, ndhF, and nrITS. International Journal of Plant Science 170: 355-364.

Kausel, E. 1949. Comentario sobre las ericáceas y epacridáceas chilenas. Revista Universitaria (Santiago) 34(I): 155-178.

Luebert, F. \& P. Pliscoff. 2006. Sinopsis bioclimática y vegetacional de Chile. Editorial Universitaria. Santiago de Chile. 316 pp.

Luteyn, J.L. 1995. Gaultheria. In: J. L. Luteyn (ed.), Flora Neotropica Monograph 66. Ericaceae Part II. The superiorovaried genera. Pp. 365-383. New York Botanical Garden. New York.

Middleton, D.J. \& C.C. Wilcock. 1990. A critical examination of the status of Pernettya Gaud. as a genus distinct from Gaultheria L. Edinburgh Journal of Botany 47(3): 291-301.

PhilipPI, R.A. 1864. Plantarum novarum chilensium. Centuriae inclusis quibusdam Mendocinis et Patagonicis. Linnaea 33: $1-308$.

ReIche, C. [K. F.]. 1905. Estudios críticos sobre la flora de Chile. Anales Univ. Chile 117: 481-517. (Flora de Chile 5:66101. 1910).

Sleumer, H. 1985. Taxonomy of the genus Pernettya Gaud. (Ericaceae). Botanische Jahrbücher für Systematik, Pflanzengeschichte und Pflanzengeographie. Leipzig 105 (4): 449-480.

Teillier, S. \& F. Escobar. 2013. Revisión del género Gaultheria L. (Ericaceae) en Chile. Gayana Botánica 70 (1): 136-153.

UICN. 2001. Categorías y criterios de la lista roja de la UICN: Versión 3.1. Comisión de Supervivencia de Especies de la UICN. UICN, Gland, Suiza y Cambridge, Reino Unido. ii +33 pp.

Recibido: 19.06.15

Aceptado: 10.03.16 